\title{
Searching for late-time hard X-ray emission of Swift GRBs
}

\author{
N. Ohmori ${ }^{* a}$, T. Sakamoto ${ }^{b}$ and M. Yamauchi ${ }^{a}$ \\ $\dagger$ \\ Author affiliation: University of Miyazaki ${ }^{a}$, Goddard Space Flight Center ${ }^{b}$ \\ E-mail: ohmorieastro.miyazaki-u.ac.jp, \\ takanoriemilkyway.gsfc.nasa.gov vamauchieastro.miyazaki-u.ac.jp
}

We report a search for the late-time hard X-ray emission from Swift GRBs by processing the BAT survey data. We are interested in investigating weak hard X-ray emission especially during the shallow decay phase in the Swift/XRT light curves, and also, coincident hard X-ray emission during the late-time flares seen by Swift/XRT. In GRB 050525A, we found significant hard X-ray emission by BAT up to 1 day after the burst in our processing. This interesting result should provide crucial information to understand afterglow emission combining other multi-wavelength data. We present the BAT survey processing results of GRB 050525A, GRB 060614, GRB 060729 and GRB 080916A.

Gamma-Ray Bursts 2012 Conference-GRB2012,

May 07-11, 2012

Munich, Germany

* Speaker.

${ }^{\dagger}$ A footnote may follow. 


\section{Introduction}

The Swift X-Ray Telescope (XRT) observed early X-ray afterglow of many gamma-ray bursts (GRBs). In some GRBs, the decay slope of the XRT light curve shows shallower than -1 . This decay slope can not be explained by a typical GRB afterglow model (Zhang et al. 2006). Furthermore, flares are occasionally seen in the late-time part of X-ray afterglow light curve. Those observational properties in the X-ray afterglow light curve might suggest that the GRB central engine is still active after the prompt gamma-ray emission is over. If this is true, the late-time hard X-ray emission might be seen by the data of the Swift Burst alert telescope (BAT). Based on this motivation, We searched for the late-time hard X-ray emission associated to Swift GRBs by processing the BAT survey data.

\section{Analysis}

We processed the BAT survey data of GRB 050525A (Foley et al. 2005), GRB 060614 (Fugazza et al. 2006), GRB 060729 (Christina et al. 2006) and GRB 080916A (Fynbo et al. 2008). The redshift of those four GRBs are less than 0.7. The Swift BAT data analysis was performed using the standard FTOOLS in the HEADAS version 6.10 package. We also used the latest calibration database. We downloaded and processed the pointing observation data of the GRBs. We make 5 channel light curve in the $14-25 \mathrm{keV}, 25-50 \mathrm{keV}, 50-100 \mathrm{keV}, 100-195 \mathrm{keV}$ and 14-195 $\mathrm{keV}$ band. Table 1 shows redshift of four GRBs.

To investigate a weak hard X-ray emission associated to a GRB in BAT, we need to understand a background behavior around the GRB at the same time. This is a crucial check because BAT is a background dominated instrument and it involves a very complicated data processing to make the sky image.

In this work, we selected five background objects near GRB and extracted light curves for those objects to check the background fluctuation during the GRB observation. Figure 1 shows the example of five background objects which we selected near GRB 050525A.

\begin{tabular}{|c|c|}
\hline GRB name & redshift $(z)$ \\
\hline GRB050525A & 0.606 \\
\hline GRB060614 & 0.1257 \\
\hline GRB060729 & 0.5428 \\
\hline GRB080916A & 0.6887 \\
\hline
\end{tabular}

Table 1:redshift for GRB name. In this work, the redshift of those four GRBs are less than 0.7.

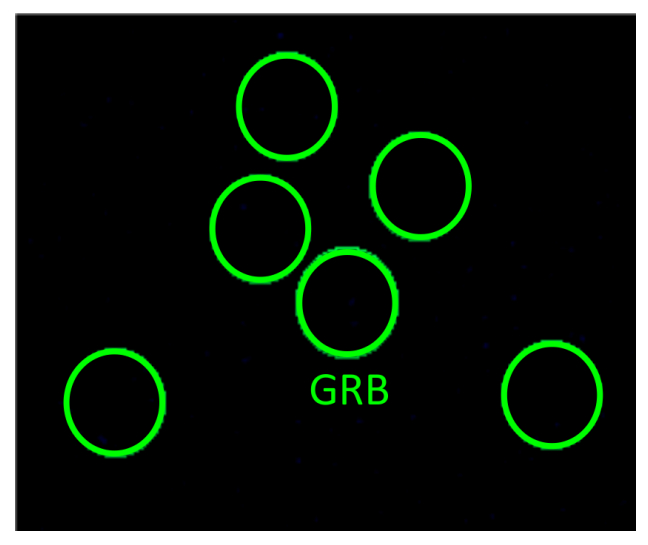

Figure 1:GRB050525A and background object position. This figure shows five background objects which we selected near GRB 050525A. 


\section{Results of GRB $050525 A$}

\subsection{Light curve of GRB $050525 \mathrm{~A}$}

Figure 2 shows the distribution of signal to noise ratio in the 14-195 keV band for GRB 050525A. And Figure 2 shows that this distribution is well fitted by a gauss function with a center of 1.081. This result indicates that the data show a systematically positive count-rate at the sky position of GRB 050525A.

Figure 3 shows GRB 050525A and three background light curves. The count rate of three background regions around the GRB 050525A location are all consistent with zero. Whereas, the light curve of GRB $050525 \mathrm{~A}$ shows a systematically positive count-rate up to $10^{5} \mathrm{sec}$ after the trigger. Therefore, a systematically positive count-rates seen in GRB 050525A by BAT is unlikely due to the systematic problem in the background.

Figure 4 shows BAT (14-195 keV) and XRT (0.3-10 keV). Continuous 1-3 sigma weak emission appears to exist up $10^{5} \mathrm{sec}$ in the BAT survey data. Whereas, the XRT light curve shows a power-law decay with the index of -1.5 .

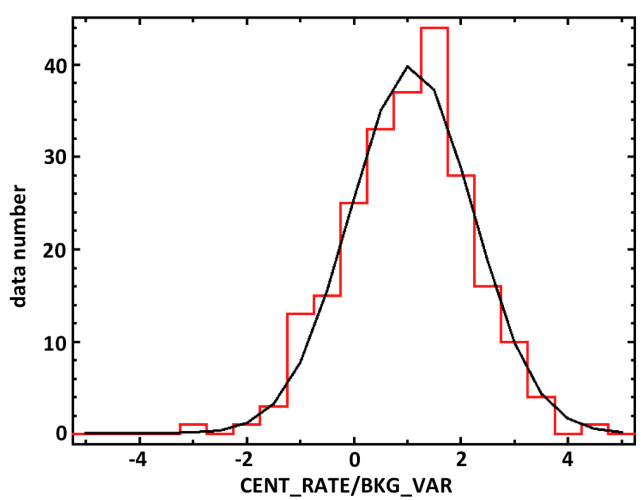

Figure 2:Distribution of signal to noise ratio (14-195 $\mathrm{keV})$. This distribution is well fitted by a gauss function with a center of 1.081 .

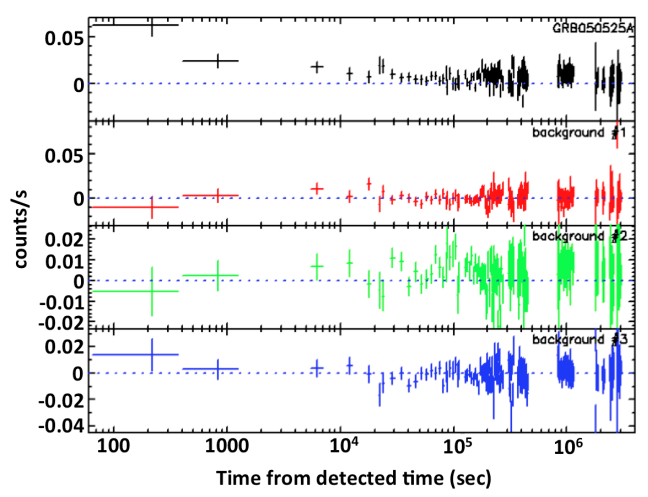

Figure 3:BAT and background light curve. From top to bottom, light curve of GRB $050525 \mathrm{~A}$ in the $14-195 \mathrm{keV}$, light curve of background 1 in the 14-195 keV, light curve of background 2 in the $14-195 \mathrm{keV}$, light curve of background 3 in the $14-195 \mathrm{keV}$.

\subsection{BAT and XRT spectrum of GRB 050525A}

Figure 5 shows $v F_{v}$ spectrum of GRB 050525A of BAT and XRT. The XRT spectrum from $T_{0}+3000 \mathrm{~s}$ to $T_{0}+10^{5} \mathrm{~s}$ is well fitted by a single power-law with absorption, where $T_{0}$ is the BAT trigger time in BAT. Table 2 summarizes XRT spectrum fitting parameter of photon index $\alpha$, hydrogen column density $N_{\mathrm{H}}$ and flux in the $0.3-10 \mathrm{keV}$ band. The BAT flux is converted from the avarage count-rate measured from $T_{0}+3000 \mathrm{~s}$ to $T_{0}+10^{5} \mathrm{~s}$ in a single power law model with a photon index of -2. Table 3 summarizes BAT parameters of BAT avaraged count rate, $\alpha$, and flux for 0.3-10 keV. As shown in Figure 4, the BAT possible late-time emission is three order of magnitude higher in flux than that of the XRT X-ray afterglow emission measured at the same time interval. If the detection of the late-time emission in BAT is real, it requires an additional spectral component to explain this possible BAT late-time hard X-ray emission. 


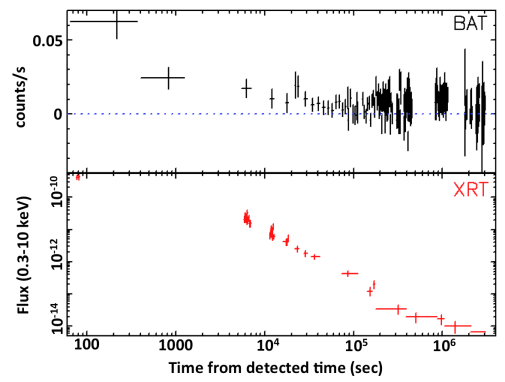

Figure 4:BAT and XRT light curve. From top to bottom, light curve of BAT in the 14-195 keV, light curve of XRT in the $0.3-10 \mathrm{keV}$.

\begin{tabular}{|c|c|}
\hline parameter & value(XRT) \\
\hline$N_{\mathrm{H}}$ & $\begin{array}{c}1.93(-0.87,+0.95) \times 10^{21} \\
\mathrm{~cm}^{-2}\end{array}$ \\
\hline Photon index & $2.10(-0.15,+0.16)$ \\
\hline Flux $(0.3-10 \mathrm{keV})$ & $\begin{array}{c}4.38(-0.38,+0.42) \times 10^{-12} \\
\mathrm{erg} / \mathrm{cm}^{2} / \mathrm{s}\end{array}$ \\
\hline
\end{tabular}

Table 2:best fit parameter and flux of XRT spectrum. The fitting model of spectrum in the $0.3-10 \mathrm{keV}$ is a single power law model with absorption.

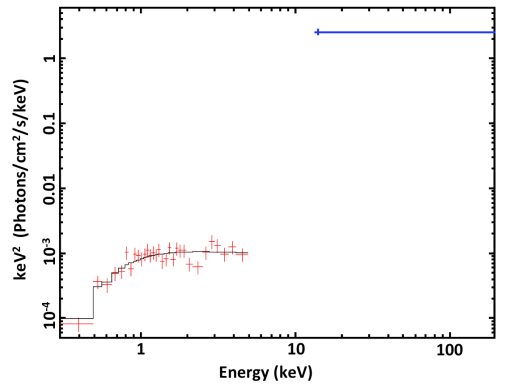

Figure 5:BAT and XRT spectrum. This figure shows spectrum of GRB 050525A of BAT and XRT from $T_{0}+3000 \mathrm{~s}$ to $T_{0}+10^{5}$.

\begin{tabular}{|c|c|}
\hline parameter & value(BAT) \\
\hline averaged count rate & $\begin{array}{c}0.007\left(T_{0}+3000 \mathrm{~s}-T_{0}+10^{5} \mathrm{~s}\right) \\
\text { count } / \mathrm{s}\end{array}$ \\
\hline Photon index & $2($ assumed $)$ \\
\hline & $4.89 \times 10^{-9}$ \\
Flux $(0.3-10 \mathrm{keV})$ & $\mathrm{erg} / \mathrm{cm}^{2} / \mathrm{s}$ \\
\hline
\end{tabular}

Table 3:best fit parameter and flux of BAT spectrum. The BAT flux is converted from the avarage count-rate.

\section{Conclusion}

Possible late-time hard X-ray emission has been found for GRB 050525A. No significant late-time hard X-ray emission was found for GRB 050826, GRB 060614, GRB 060729 and GRB 080916A. The analysis to search for a hard X-ray emission of complete Swift known-z GRB samples is in progress.

The hard X-ray flux of the possible late-time emission of GRB 050525A is three times higher than the X-ray afterglow flux observed by XRT. Therefore, an additional spectral component is required to explain the BAT late-time emission.

Our search suggests that a relatively bright late-time hard X-ray emission associated with a GRB might be a common. A GRB afterglow observation in hard X-rays by near future missions which carries a high sensitive hard X-ray telescope such as NuStar and Astro-H should shed a new light on understanding a GRB late-time emission.

\section{References}

[1] Christina C. T., et al., GRB 060729 redshift, GCN Circ., 5373, 2006, http://gcn.gsfc.nasa.gov/gen3/5373.gcn3

[2] Foley. R. J., et al., GRB 050525a: Gemini/GMOS Spectra, GCN Circ., 3483, 2005, http://gcn.gsfc.nasa.gov/gen3/3483.gcn3

[3] Fugazza. D., et al., GRB 060614: redshift confirmation, GCN Circ., 5276, 2006, http://gcn.gsfc.nasa.gov/gen3/5276.gcn3

[4] Fynbo. J. P. U., et al., GRB080916A: Spectroscopic redshift, GCN Circ., 8254, 2008, http://gcn.gsfc.nasa.gov/gen3/8254.gcn3

[5] Zhang. B., et al., Physical Processes Shaping Gamma-Ray Burst XRay Afterglow Light Curves: Theoretical Implications from the Swift X-Ray Telescope Observations, ApJ, 642, 2006, 354 